\section{Extraarticular Manifestations of Rheumatoid Arthritis in Patients under Anti-tumor Necrosis Factor- $\alpha$ Treatment}

\section{To the Editor:}

We read with great interest the article on extraarticular manifestations of rheumatoid arthritis (RA) developing in patients under anti-tumor necrosis factor- $\alpha(\mathrm{TNF}-\alpha)$ treatment by Negoescu, et al ${ }^{1}$. These manifestations were present in $42 / 152$ patients $(27.6 \%)$, of whom $22 / 152(14.5 \%)$ were newly diagnosed and 5/152 (3.3\%) were worsened preexisting manifestations of lung disease, subcutaneous rheumatoid nodules, and cutaneous vasculitis. One patient had stable chronic renal failure of unclear cause. Conversely, regression of cutaneous nodules was observed in 4 patients, and some patients were in disease remission while others had active disease. This paper raises serious concern for physicians prescribing these biotherapeutic agents, and its results require clarification.

First, with regard to the lung lesions, a wide variety of lung diseases have been described in patients with RA including interstitial lung disease (ILD) and rheumatoid nodules, but also pleural disease, Caplan syndrome, and bronchiectasia ${ }^{2}$. What were the features of lung lesions in patients in this series? How was the diagnosis established? Was an adverse reaction because of other drugs ruled out? Exacerbation and appearance of ILD were reviewed in a large series of 122 patients receiving anti-TNF- $\alpha$ agents ${ }^{3}$. Withdrawal of biologic agents resulted in complete resolution, partial resolution, and no resolution in $40 \%, 25 \%$, and $35 \%$ of patients, respectively. Of note, $29 \%$ of them died during the followup, and $70 \%$ during the first 5 weeks after initiating biologic therapy. Risk factors for death were age $>65$ years, later onset of ILD, previous treatment with immunosuppressive drugs, and previous diagnosis of ILD. In another study, 12 patients treated with etanercept who developed ILD were reviewed, 7 had preexisting ILD, 10 patients recovered at the end of etanercept treatment, but 2 of them died 4 . Therefore, how severe was the "lung disease" in the patients of the present study? Of interest, noninfectious pulmonary complications consisting of ILD, idiopathic pulmonary fibrosis, and allergic pneumonitis, and culture-negative pneumonia have been also described under newer biological agents such as rituximab, certolizumab, golimumab, tocilizumab, and abatacept ${ }^{5}$. Underlying mechanisms are still disputed. However, we can mention that the inflammatory injury is Th1-mediated in RA. Conversely, a Th2 pathway is advocated in the genesis of ILD. TNF- $\alpha$ blockade could result in cell lysis with release of macrophage-derived proteolytic enzymes injuring the lung epithelium and potentiating the fibrotic cascade, resulting in pulmonary fibrosis ${ }^{3}$. In the same way, development of rheumatoid nodules of the lung has been reported in patients receiving anti-TNF- $\alpha$ agents ${ }^{6,7,8}$. Indeed, rheumatoid nodules can involve skin, but also other sites such as lung, spine, heart valve, and gastrointestinal tract. In rheumatoid nodules (whatever their sites and in inflamed synovium), expression of TNF- $\alpha$ has been demonstrated. Development of nodules under anti-TNF- $\alpha$ agents indicates that other cytokines (transforming growth factor $\beta$ and interleukin 1) could play a role in granuloma formation. The differential diagnosis of pulmonary nodulosis is wide, including malignancies, infections (tuberculosis, histoplasmosis, coccidioidomycosis), and sarcoidosis. Therefore, we consider that a definite diagnosis of the pulmonary lesions is mandatory.

With regard to cutaneous lesions of RA, these lesions consist in specific manifestations such as rheumatoid nodules (and close conditions such as accelerated rheumatoid nodulosis and rheumatoid nodulosis) and nonspecific lesions including vasculitis and neutrophilic dermatoses, based on clinical and histopathological features ${ }^{9}$. Therefore, was the diagnosis of rheumatoid nodule and cutaneous vasculitis established histologically? Indeed, Negoescu, et al indicated in their study that excision of nodules was performed only in 5 patients. Moreover, was vasculitis related to rheumatoid vasculitis or secondary to an infection or to another drug? Finally, we acknowledge that firm, movable subcutaneous nodules developing on the extensor surface of the elbow, olecranon, and extensor tendons of the hands (areas prone to repetitive trauma) in patients with RA are very likely related to rheumatoid nodules ${ }^{9}$. However, these lesions should be distinguished from fibromas, xanthomas, subcutaneous granuloma annulare, sarcoidosis, gouty tophi, synovial and epidermoid cysts, and histoplasmosis. Nodule biopsy would have ascertained the diagnosis of rheumatoid nodules. Interestingly, development of rheumatoid nodules has been already reported following etanercept ${ }^{10}$. Paradoxically, TNF- $\alpha$ proinflammatory cytokine is found in immunohistochemical analysis of rheumatoid nodule biopsy, and anti-TNF- $\alpha$ therapy should be an effective treatment of these extraarticular manifestations. The pathogenesis of this paradoxical event is still disputed, but other cytokines than TNF- $\alpha$ should play a role as indicated previously.

Development or worsening of extraarticular manifestations of RA under anti-TNF- $\alpha$ agents seems to be a common phenomenon. Physicians should be aware of this possible side effect of these drugs and wonder whether these manifestations are drug-induced or an alarm of illness progression or an unrelated event. Indeed, we agree with the authors that further clinical and biological studies are required to explain this embarrassing association.

CLAUDE BACHMEYER, MD; CHRISTOPHER REIN, MD; SOPHIE GEORGIN-LAVIALLE, MD, PhD, Department of Internal Medicine, Tenon Hospital (AP-HP), Paris, France. Address correspondence to Dr. C. Bachmeyer, Department of Internal Medicine, Tenon Hospital (AP-HP), 4 rue de la Chine, 75020 Paris, France.

E-mail: claude.bachmeyer@tnn.aphp.fr

\section{REFERENCES}

1. Negoescu AF, Tennekone D, Soljak MA, Abraham SM. Extraarticular manifestations of rheumatoid arthritis develop in patients receiving anti-tumor necrosis factor- $\alpha$ Treatment: a retrospective chart review from a UK center. J Rheumatol 2014;41:1944-7.

2. Cavagna L, Monti S, Grosso V, Boffini N, Scorletti E, Crepaldi G, et al. The multifaceted aspects of interstitial lung disease in rheumatoid arthritis. Biomed Res Int 2013;2013:759760.

3. Perez-Alvarez R, Perez-de-Lis M, Diaz-Lagares C, Pego-Reigosa JM, Retamozo S, Bove A, et al. Interstitial lung disease induced or exacerbated by TNF-targeted therapies: analysis of 122 cases. Semin Arthritis Rheum 2011;41:256-64.

4. Horai Y, Miyamura T, Shimada K, Takahama S, Minami R, Yamamoto M, et al. Etanercept for the treatment of patients with rheumatoid arthritis and concurrent interstitial lung disease. J Clin Pharm Ther 2012;37:117-21.

5. Hadjinicolaou AV, Nisar MK, Bhagat S, Parfrey H, Chilvers ER, Ostör AJ. Non-infectious pulmonary complications of newer biological agents for rheumatic diseases - a systematic literature review. Rheumatology 2011;50:2297-305.

6. Kekow J, Welte T, Kellner U, Pap T. Development of rheumatoid nodules during anti-tumor necrosis factor alpha therapy with etanercept. Arthritis Rheum 2002;46:843-4.

7. Scrivo R, Spadaro A, Iagnocco A, Valesini G. Appearance of rheumatoid nodules following anti-tumor necrosis factor alpha treatment with adalimumab for rheumatoid arthritis. Clin Exp Rheumatol 2007;25:117.

8. van Ede A, den Broeder A, Wagenaar M, van Riel P, Creemers MCW. Etanercept-related extensive pulmonary nodulosis in a patient with rheumatoid arthritis. J Rheumatol 2007;34:1590-2.

9. Sayah A, English JC 3rd. Rheumatoid arthritis: a review of the cutaneous manifestations. J Am Acad Dermatol 2005;53:191-209.

10. Mackley CL, Ostrov BE, Ioffreda MD. Accelerated cutaneous nodulosis during infliximab therapy in a patient with rheumatoid arthritis. J Clin Rheumatol 2004;10:336-8.

J Rheumatol 2015;42:6; doi:10.3899/jrheum.141273 